\title{
Review of Antarctic astronomy
}

\author{
John W. V. Storey \\ School of Physics, University of New South Wales, \\ Sydney, NSW 2052, Australia \\ email: j.storey@unsw.edu.au
}

\begin{abstract}
Astronomers have always sought the best sites for their telescopes. Antarctica, with its high plateau reaching to above 4,000 metres, intense cold, exceptionally low humidity and stable atmosphere, offers what for many forms of astronomy is the ultimate observing location on this planet. While optical, infrared and millimetre astronomers are building their observatories on the ice, particle physicists are using the ice itself as a detector and exploration of the terahertz region is being conducted from circumpolar long-duration balloons. Remarkable astronomical discoveries are already coming out of Antarctica, and much, much more is just around the corner.
\end{abstract}

Keywords. Antarctica, Arctic

\section{Introduction: why Antarctica?}

Looking at Antarctica from a human perspective it may seem that this would be the last place on earth to build a astronomical observatory. Mention "Antarctica" and the horrible prospect of slowly freezing or starving to death may well come to mind. However, from an astronomical perspective, it also happens to be one of the best places on earth to build an observatory. And, thanks to a century of development in logistics capability, anyone can now work there. Like the air and the ocean, Antarctica is unforgiving of the foolhardy or unprepared. However, with several countries now operating well-established stations and providing excellent transport capabilities, anyone who loves the continent can now live and work there in safety.

Relative to the best temperate sites:

- The infrared sky is 20-50 times darker

- The water vapour content is $\sim 10$ times lower

- The free-atmosphere seeing is $\sim 2$ times better

- The coherence volume is 10-100 times greater

- The scintillation noise is $\sim 2.5$ times less

- The atmospheric boundary layer is only 10-20 metres high

- The aerosol content is 50 times lower

- The sky is continuously dark for months

For particle physicists, the vast volumes of the purest ice on earth provide an unparalleled medium for detecting neutrinos, muons, and other subatomic beasties. For folk wishing to observe above (most of) the atmosphere, the circumpolar vortex invites longduration ballooning and indeed ultra-long duration ballooning. Antarctica is not only beautiful, it is the only place on earth set aside for science by international treaty.

There are also of course some disadvantages and discouragements for the Antarctic astronomer:

- It is exceedingly cold. However, this from a human, not an engineering perspective. It is not as cold as the insides of a dewar, nor indeed space. A competent engineer can design for any temperature. While "cold" might sound daunting, this is probably the least difficult issue to cope with. 
- There is longer twilight. The sun simply spends more time close to the horizon than at temperate sites. This can be good or bad, depending on the science, but is usually bad.

- The sky coverage is less. This is the flip-side of the fact that those objects that are visible are generally continuously visible.

- The relative humidity is high. This is perhaps surprising - the absolute humidity is lower than that in a cylinder of commercial dry nitrogen. However, given the intense cold, objects close to the ground are susceptible to slowly icing up if they are allowed to fall below (or even sit at) ambient temperature.

- The temperature in the boundary layer is unstable. This presents an engineering challenge to the realisation of the exception image quality that the site can potentially deliver. "Mirror seeing" must be avoided, and this has traditional required that the mirror temperature closely track the ambient temperature.

- At optical wavelengths, aurorae are at best a nuisance and, for some science, potentially a strong disadvantage.

- The accessibility is less than ideal, as even the strongest advocate for Antarctica would have to admit. Still, it beats L2.

This brings us to the inevitable comparison with space. Why bother with Antarctica, when space invariably offers even better observing conditions? There are two principal reasons: deployment to Antarctica is one thousand times cheaper than space $(\sim \$ 10 / \mathrm{kg}$ versus $\sim \$ 10,000 / \mathrm{kg}$ ) and - whatever your views are on spending several hours in a Hercules $\mathrm{C} 130$ - it is much more accessible. Experiments can be developed and deployed quickly, and can be maintained and upgraded on annual basis during the summer months.

An extensive review of Antarctic astronomy was published by Michael Burton just two years ago (Burton 2010), so in this introductory paper to the Symposium only the briefest of brief overviews of the field will be given. In mentioning various experiments, no attempt is made to be comprehensive: I have simply chosen a few illustrative examples to whet the reader's appetite for these proceedings.

\section{The existing stations}

Antarctica is large - roughly 50\% larger than China, Europe, or the USA. It should therefore not be surprising that the climates and, with them, the observing conditions, are very different at the different sites. Most astronomical work has been carried out from the inland stations, as these are higher, drier, and calmer than the coast.

\subsection{South Pole}

First established by the USA in 1957, the Amundsen-Scott Station at the geographic south pole has been host to a wide range of highly successful telescopes. It is a unique site, offering continuous observations of celestial objects at constant zenith distance. Although true geostationary satellites are not visible from South Pole (nor indeed any site south of about $-80^{\circ}$ latitude, the National Science Foundation is nevertheless able to provide high bandwidth communications through "wobbly" geostationary satellites and through TDRSS.

Several major cosmic microwave background experiments at South Pole take advantage of the unique location. These include the imaginatively-named South Pole Telescope, a 10 metre diameter sub-millimetre telescope (Carlstrom et al. 2011) that has carried out extensive surveys using the Sunyaev-Zel'dovich effect.

Particle experiments at South Pole take advantage of the massive quantities of pure ice to detect neutrinos and muons. The largest of these is Ice Cube (Ahrens et al. 2004), 
and several new experiments (eg., the Askaryan Radio Array, Hanson et al. 2012) are under development.

\subsection{McMurdo}

Sited on the coast, the US McMurdo station is generally uncompetitive as an astronomical site. Nevertheless, it has proved to be an ideal location from which to launch high altitude balloons, which float around the continent and, after a period of typical 14 days, often return more or less directly overhead again. A recent well-publicised example is BLAST (Devlin et al. 2009).

\subsection{Dome A}

At an elevation of 4,050 metres, Dome $\mathrm{A}$ is the highest point on the plateau. Kunlun station was established there by China in 2008. Although only inhabited over a few weeks each summer, Kunlun hosts experiments that operate year-round using an autonomous power, heat and communications facility called PLATO (Yang et al. 2009).

Data from Kunlun indicate that the site offers exceptionally good terahertz transmission (Yang et al. 2010) and, of particular importance for optical/infrared astronomy, a boundary layer thickness of typically less than 14 metres (Bonner et al. 2010). The CSTAR wide-field optical telescopes have operated robotically at Kunlun for several years now, and have returned a wealth of data (Zhou et al., 2010, Wang et al. 2011).

\subsection{Dome $C$}

Completed in 2005, the French/Italian Concordia station at Dome C offers extremely good free-atmosphere seeing (eg., Lawrence et al. 2004, Aristidi et al. 2005, Giordano et al. 2012), a low boundary layer and very good cloud-cover statistics (Crouzet et al. 2010). The site hosts several important astronomical facilities, including the wide-field optical survey telescope ASTEP-400 (Crouzet et al. 2011).

At millimetre wavelengths, Battistelli et al. (2012) have used the BRAIN Pathfinder Experiment to study the atmospheric transmission and polarisation properties, prior to deployment of a planned interferometer to study the cosmic microwave background.

Dome C also offers very favourable conditions for solar astronomy, with very low atmospheric scattering (eg., Faurobert et al. 2012).

\subsection{Dome F}

The Japanese station at Dome F (Dome Fuji) is an another outstanding observing site. Although it operated as a year-round station for a number of years, it currently is open only during the summer. As with Dome A, autonomous power systems will be used - at least for the near future - to allow instruments to operate through the winter months. A $40 \mathrm{~cm}$ infrared telescope is planned for installation there at the end of 2012 (Okita et al. 2010).

\subsection{Ridge $A$}

Modelling of site conditions in Antarctica has been carried out by a number of researchers (eg., Lascaux et al. 2011), and has the advantage that predictions can be made that cover longer periods and a greater range of locations than are available from in situ observational data. By analysing available site-testing, satellite and meteorological data and applying appropriate models, Saunders et al.(2009) set out to determine what observing conditions would be like across the entire Antarctic plateau. Their conclusion was that there was no unique "best" site, and that each of the existing stations offered a particular set of advantages. However a new location, dubbed Ridge A, was predicted to not only 
have the lowest precipitable water vapour (and hence best $\mathrm{THz}$ transmission) but also to be an exceptionally good site on all criteria.

To test this hypothesis, and and the same time take advantage of the $\mathrm{THz}$ windows opened up, a robotic $800 \mathrm{GHz}$ telescope was deployed there at the beginning of 2012 . Initial indications (Kulesa 2012) are that Ridge A (and, by implication, other high plateau sites) offers unparalleled opportunities for observations in this hitherto inaccessible part of the spectrum.

\section{New projects}

Some sense of the breadth and depth of Antarctic astronomy can be gauged from the large number of major new telescopes that are either under construction or in the advanced planning stages. These include AFSIIC, AIRT-40, ARA, AST3, DATE5, EBEX, IRAIT, KDUST, PILOT, POLAR, QUBIC and Super-TIGER, and there are many more!

\section{The Arctic}

While Antarctica is a continent surrounded by ocean, the Arctic is an ocean surrounded by continents. The north pole is typically at an elevation of only a few metres above sea level, and is thus not an attractive site for astronomy. However, close to the north pole are several sites that offer many of the advantages of their Antarctic counterparts. For example Summit station on Greenland is at the same elevation as Dome C, and at the same (absolute) latitude. A 12 metre dish is to be installed there over the coming year for use in sub-millimetre interferometery.

Site testing in the Arctic has focussed on Summit, and at sites on Ellesmere Island in northern Canada. Both locations appear to have superb potential. On Ellesmere Island, site studies over the past few years (Steinbring et al.(2012)) have demonstrated excellent conditions for optical astronomy.

\section{Conclusion}

With this symposium, Antarctic astronomy has come of age. The scientific results now routinely coming from Antarctic observatories demonstrate not only the unique qualities of that continent, but also that it offers a practical and cost-effective way to conduct science that would be impossible, or much more expensive, to conduct elsewhere.

In future IAU meetings, Antarctic astronomy might no longer be seen as so obviously "different". Major scientific results from Antarctica are now having an impact in many different fields, and gain their prominence not by being "Antarctic", but by the pivotal contributions they make to our understanding of astronomy. May such science continue to flow from Antarctica, and continue to bear witness to the advantages of observing from this beautiful, unique continent.

\section{References}

Ahrens, J., Bahcall, J. N., Bai, X., et al. 2004, New Astr., 48, 519

Aristidi, E., Agabi, A., Fossat, E., Azouit, M., Martin, F., Sadibekova, T., Travouillon, T., Vernin, J., et al. 2005, A $\mathscr{G} A, 444,651$

Battistelli, E. S., Amico, G., Baù, A., et al. 2012, MNRAS, 423, 1293

Burton, M. G. 2010, A\&A Rev., 18, 417

Carlstrom, J. E., Ade, P. A. R., Aird, K. A., et al. 2011, PASP, 123, 568 
Crouzet, N., Guillot, T., Agabi, A., Rivet, J., Bondoux, E., et al. 2010, A\&\&A, 511, 36

Crouzet, N., Guillot, T., Agabi, K., Daban, J.-B., Abe, L., Mekarnia, D., Rivet, J.-P., FanteCaujolle, Y., Fressin, F., Gouvret, C., Schmider, F.-X., Valbousquet, F., Blazit, A., Rauer, H., Erikson, A., Fruth, T., Aigrain, S., Pont, F., \& Barbieri, M. 2011, EPJ Web of Conferences, 11, 06001

Devlin, M. J., Ade, P. A. R., Aretxaga, I., et al. 2009, Nature, 458, 737

Faurobert, M., Arnaud, J., \& Vernisse, Y. 2012, EAS Publications Series, 55, 365

Giordano, C., Vernin, J., Chadid, M., Aristidi, E., Agabi, A., \& Trinquet, H. 2012, PASP, 124, 494

Hanson, K., ARA Collaboration 2012, Journal of Physics Conference Series, 375, 052037

Kulesa, C. 2012, private communication

Lascaux, F., Masciadri, E., \& Hagelin, S. 2011, MNRAS, 411, 693

Lawrence, J. S., Ashley, M. C. B., Tokovinin, A., \& Travouillon, T. 2004, Nature, 431, 278

Okita, H., Ichikawa, T., Yoshikawa, T., Lundock, R. G., \& Kurita, K. 2010, Proc. SPIE, 7733,

Saunders, W., Lawrence, J. S., Storey, J. W. V., et al. 2009, PASP, 121, 976

Steinbring, E., Ward, W., \& Drummond, J. R. 2012, PASP, 124, 185

Wang, L., Macri, L. M., Krisciunas, K., et al. 2011, AJ 142, 155

Yang, H., Allen, G., Ashley, M. C. B., et al. 2009, PASP, 121, 174

Yang, H., Kulesa, C. A., Walker, C. K., Tothill, N. F. H., Yang, J., Ashley, M. C. B., Cue, X., Feng, L., Lawrence, J. S., Luong-Van, D. M., Storey, J. W. V., Wang, L., Zhou, X., \& Zhu, Z. 2010, PASP, 122, 490

Zhou, X., Fan, Z., Jiang, Z., et al. 2010, PASP, 122, 347 